\title{
Revealing Particle Growth Mechanisms by Combining High-Surface-Area Catalysts Made with Monodisperse Particles and Electron Microscopy Conducted at Atmospheric Pressure
}

\author{
Shuyi Zhang ${ }^{1,2}$, Matteo Cargnello ${ }^{3,4}$, Wei Cai ${ }^{1}$, Christopher B. Murray ${ }^{3,5}$, George W. \\ Graham $^{1, *}$, and Xiaoqing Pan ${ }^{1,2,6, *}$ \\ ${ }^{1}$ Department of Materials Science and Engineering, University of Michigan, Ann Arbor, MI 48109, USA \\ ${ }^{2}$ Department of Chemical Engineering and Materials Science and ${ }^{6}$ Department of Physics and Astronomy, \\ University of California - Irvine, Irvine, CA 92697, USA \\ ${ }^{3}$ Department of Chemistry and ${ }^{5}$ Department of Materials Science and Engineering, University of \\ Pennsylvania, Philadelphia, PA 19104, USA \\ ${ }^{4}$ Department of Chemical Engineering and SUNCAT Center for Interface Science and Catalysis, Stanford University, \\ Stanford, CA 94305, USA \\ *Corresponding Authors, Email: xiaoqing.pan@uci.edu, gwgraham@umich.edu
}

\begin{abstract}
:
In-situ aberration-corrected transmission electron microscopy, conducted in forming gas $\left(5 \% \mathrm{H}_{2} / \mathrm{N}_{2}\right)$ at atmospheric pressure and elevated temperatures (up to $800^{\circ} \mathrm{C}$ ), was used to follow the growth of $\mathrm{Pt}$ nanoparticles dispersed on a high-surface-area $\mathrm{Al}_{2} \mathrm{O}_{3}$ support. These direct observations, facilitated by the use of monodisperse Pt nanoparticles, allowed for an unambiguous separation of the relative contributions of Ostwald ripening and particle migration/coalescence to the overall growth process and made possible the estimation of corresponding energetic and kinetic parameters. Whereas the parameters describing Ostwald ripening were as expected, the diffusion coefficient characterizing particle migration was surprisingly small. Size-selection methods were thus shown to have significant potential for suppressing Ostwald ripening, the dominant growth process, in practical catalysts.
\end{abstract}


Keywords: In-situ Electron Microscopy, Mono-Dispersed Pt nanoparticles, Ostwald

Ripening, Particle Migration and Coalescence, High-Surface-Area $\mathrm{Al}_{2} \mathrm{O}_{3}$

\section{INTRODUCTION}

Supported transition metal nanoparticles (NPs) find widespread applications in catalysts, ${ }^{1-5}$ sensors, ${ }^{6,7}$ fuel cells, ${ }^{8-10}$ and several other energy conversion devices. ${ }^{11,12}$ In many of these applications, stable materials that sustain high temperatures without losing active surface area are required. This need is particularly important when precious metals are used, due to their dwindling supply and increasing demand that require NP growth over time to be mitigated as much as possible. The understanding of mechanisms involved in the growth of supported metal NPs is thus crucial, and despite the large body of work, still not complete. In particular, a detailed atomistic description of the processes taking place under conditions that are closest to the final applications is still missing.

The ability to clearly differentiate between the two most commonly mentioned deactivation phenomena, Ostwald ripening (OR) and particle migration and coalescence (PMC), is important to the design of more stable catalysts. Difficulty arises from the fact that samples in which particle size distributions are broad do not easily allow for discrimination between the two mechanisms, in which on one side single atom species participate in the particle growth (OR), and on the other the entire particles merge to produce larger ones (PMC).

Various observation techniques have been exploited in this sense. Indirect techniques, such as chemisorption or X-ray diffraction, typically applied to large ensembles of NPs, provide only averaged information about particle size distributions (PSDs). Direct measurement of PSDs by transmission electron microscopy (TEM), on the other hand, is plagued by the uncertainty of conclusions drawn from severely limited sample size in highly heterogeneous samples. Further, the validity of using the shape of the PSD to discriminate between the two main growth processes, has been questioned because similar log-normal distributions can satisfactorily fit both mechanisms of growth. ${ }^{13}$ Efforts to characterize these processes on model supports using the environmental TEM, which allows for real-time observation, have been reported and greatly advanced the fundamental understanding, ${ }^{14-18}$ but the material and pressure gaps inherent in such work still raise questions about whether the results hold true for conditions closer to realistic applications.

In the present study, we employ a recently developed in-situ TEM technique, ${ }^{19-21}$ which can be used at atmospheric pressure, to identify the separate contributions of OR and $\mathrm{PMC}$ to the growth of mono-dispersed Pt NPs in high-surface-area $\mathrm{Pt} / \mathrm{Al}_{2} \mathrm{O}_{3}$ catalysts under reducing conditions. The use of this technique on realistic samples eliminates both pressure and material gaps, thus allowing us to directly observe both processes separately in real time. Further, the use of mono-dispersed Pt NPs not only allows for the suppression of OR, as recently shown on a model planar support, ${ }^{22}$ but also demonstrates the utility of this approach for controlling growth rates of supported metal NPs for potentially realistic applications. 


\section{EXPERIMENTAL}

\section{Preparation of $\mathrm{Pt} / \mathrm{Al}_{2} \mathrm{O}_{3}$ samples}

Pt nanocrystals were synthesized by following established procedure with minor modifications. ${ }^{5}$ All syntheses were performed using standard Schlenk techniques. $\mathrm{Pt}(\mathrm{acac})_{2}(98 \%$, Acros Organics) was used as metal precursor. Trioctylamine (TOA, 97\%) was purchased from Acros. Oleylamine (OLAM, 70\%), trioctylphosphine (TOP, 97\%) and oleic acid (OLAC, 90\%) were purchased from Sigma-Aldrich and used as received. $0.2 \mathrm{mmol}$ of $\mathrm{Pt}(\mathrm{acac})_{2}$ were mixed with oleylamine $(2 \mathrm{mmol})$, oleic acid $(8 \mathrm{mmol})$ in TOA $(10 \mathrm{~mL})$ and evacuated at RT for 5 minutes. Trioctylphosphine $(0.1 \mathrm{mmol}$ for smaller particles, $0.5 \mathrm{mmol}$ for larger ones) was then added and the mixture evacuated further and heated to $120{ }^{\circ} \mathrm{C}$ for 30 minutes. The flask was flushed with nitrogen and heated very quickly $\left(\sim 40{ }^{\circ} \mathrm{C} \mathrm{min}^{-1}\right)$ to $250{ }^{\circ} \mathrm{C}$ (smaller particles) or $350{ }^{\circ} \mathrm{C}$ (larger particles). After 30 minutes of reaction under continuous stirring, the solution was cooled to room temperature. The particles were isolated by addition of isopropanol and centrifugation ( $8000 \mathrm{rpm}, 3$ minutes). No size selection procedures were employed. The particles were soluble in low-polar solvents (hexanes, toluene, chloroform, THF) and were finally dissolved in toluene or hexanes for further analysis. It was found that a small volume of OLAM $(50 \mu \mathrm{L})$ was generally needed to ensure the complete redissolution of the particles.

The high surface area alumina (Sasol TH100-150) was calcined to $900{ }^{\circ} \mathrm{C}$ for $24 \mathrm{~h}$, and therefore stable under the condition utilized in our study. Stock solutions at known concentrations of Pt were prepared in hexanes and slowly added to a mixture of $200 \mathrm{mg}$ of calcined alumina powder well dispersed in toluene $(15 \mathrm{~mL})$ by sonication. Adsorption of the particles occurs instantaneously, and the powder is isolated by centrifugation and dried at $120{ }^{\circ} \mathrm{C}$ overnight. Samples were prepared with final nominal Pt loading of 0.5 $\mathrm{wt} \%(0.25 \mathrm{wt} \%$ for each size in the mixed-size sample). Removal of the ligands was performed on the dried samples by following a fast thermal treatment at $700{ }^{\circ} \mathrm{C}$ for $30 \mathrm{~s}$ as recently reported. ${ }^{23}$

\section{Microscopy characterization}

In-situ observations were carried out with a double spherical aberration-corrected microscope (JEOL JEM-3100R05) operated at $300 \mathrm{kV}$ in combination with a closed gas cell system (Protochips Atmosphere ${ }^{\mathrm{TM}}$ ), as recently adopted in another in-situ study. ${ }^{20}$ Further experimental details, including the procedure used for measuring NP areal densities, are in the Supporting Information (SI).

\section{RESULTS AND DISCUSSION}

Pt NPs, either $2.2 \pm 0.4 \mathrm{~nm}$ (referred to as "small") or $4.4 \pm 0.2 \mathrm{~nm}$ (referred to as "large") in diameter, or a mixture thereof (referred to as "mixed") were prepared and supported on high-surface-area alumina. Based on alumina surface area and the nominal Pt loading, the expected NP areal density was $\sim 10^{-3} \mathrm{~nm}^{-2}$ in the small and mixed samples and roughly ten times smaller in the large sample, though local variations in particle density were found in some regions of the sample. For the purposes of this study, regions with comparable densities were selected for detailed observation. The measured average areal density of NPs for the small, large and mixed samples analyzed below was $12.6 \times 10^{-4} \mathrm{~nm}^{-2}, 6.9 \times 10^{-4}$ $\mathrm{nm}^{-2}$ and $10.6 \times 10^{-4} \mathrm{~nm}^{-2}\left(7.9 \times 10^{-4} \mathrm{~nm}^{-2}\right.$ for NPs smaller than $\left.3 \mathrm{~nm}\right)$, respectively. A low- 
magnification high-angle annular dark field (HAADF) image of the as-synthesized mixed NP sample is shown in Figure 1(a). (Corresponding images of small and large NP samples are shown in the Figure S3.) Atomic resolution HAADF images of individual large and small Pt NPs are shown in Figures 1(b) and (c). These images were taken $e x$ situ, with the mixed NP sample supported on a carbon grid.

Samples were heated under 760 Torr of forming gas $\left(5 \mathrm{vol} \% \mathrm{H}_{2}\right.$ in $\left.\mathrm{N}_{2}\right)$ at $600{ }^{\circ} \mathrm{C}, 700$ ${ }^{\circ} \mathrm{C}$, and $800{ }^{\circ} \mathrm{C}$, for $5 \mathrm{~h}$ at each temperature, inside the gas cell in the microscope. Sequential images of 6-10 different areas in each sample were taken 4-5 times at each temperature. These images were taken with an electron beam diameter about $0.1 \mathrm{~nm}$ and a probe current of 10pA (the spreading of the probe due to the $\mathrm{SiN}$ window is not accounted), and the beam was valved off between image acquisitions, in order to avoid possible beam effects, as detailed in SI. Histograms of PSDs measured from such images at the conclusion of the treatments at 700 and $800{ }^{\circ} \mathrm{C}$ are shown in Figure 2, together with the initial PSDs that were measured at $300{ }^{\circ} \mathrm{C}$. As shown in Figure 2(a), no significant change occurred in the PSD of the small NP sample until $800^{\circ} \mathrm{C}$, after which the mean particle size had increased by $0.5 \mathrm{~nm}, \sim 23 \%$. By contrast, the PSD of the large NP sample remained basically unaffected throughout the entire experiment, as shown in Figure 2(b). The largest change in PSD, by far, occurred in the mixed NP sample, where most of the small NPs disappeared during the $5 \mathrm{~h}$ at $800{ }^{\circ} \mathrm{C}$, as shown in Figure 2(c). It is important to emphasize that our initial (and final, when stationary) PSDs are truly representative of the entire sample due to the high uniformity of the particles, a situation which has been exploited in previous work. ${ }^{5}$ The large change between the initial and final PSDs in the mixed NP sample, compared with the others, thus strongly suggests that OR has been drastically limited through size selection. Dynamic observation in real time, however, is needed for undisputable evidence of the NP growth mechanisms.

A video (shown in SI) and sequential HAADF images, such as those shown in Figure 3, provide direct evidence of the disappearance of the small NPs in the mixed sample. The artifact induced by high dose electron beam and the procedures to avoid them are detailed in SI. An example of the disappearance of a NP is indicated by the red arrow in Figure 3, and an example of the shrinking of another NP is indicated by the yellow arrow. It is important to note that the shrinking particles in Figure 3 and video in SI are representative of the small particles in the mixed sample. A plot showing the changes in the particle size (radius) as a function of time for four NPs, two shrinking and two disappearing, is shown in the bottom left panel of Figure 3. Even though particle migration was also observed in the case of some small NPs (but not in the case of larger ones shown in the video in SI), at the current particle densities the distance between particles ensures that PMC is almost completely suppressed (see also below). Thus, OR has to be the dominant mechanism of NP growth in the mixed NP sample under our conditions of NP density and environment.

The process of OR involves detachment of atoms from NPs, Fickian diffusion on the support, and deposition onto a different NP. ${ }^{24}$ Because the energy barrier for detachment of a single Pt atom from a NP onto the alumina support $(\sim 2.5 \mathrm{eV})$ is much higher than the diffusion barrier of Pt atoms on alumina (0.2-0.5 eV), we employ Equation 1 in the interface-transfer-limiting regime, as in previous work, ${ }^{17,25}$ in order to analyze our observations: 
$\frac{d r}{d t}=\frac{K}{r}\left[\exp \left(\frac{R}{r^{*}}\right)-\exp \left(\frac{R}{r}\right)\right]$

where

$K=\frac{2}{a} \frac{v_{p} \Omega \sin \theta}{\left(2-3 \cos \theta+\cos \theta^{3}\right)} \exp \left(-\frac{E_{O R}}{k_{B} T}\right)$

and

$R=\frac{2 \gamma \Omega}{k_{B} T}$

Here $r$ is the radius of a given NP at time $t$, and $r^{*}$, known as the critical radius, is the equilibrium value, below which particles shrink and above which they grow. $R$, called capillary length, is in the range of 2.8-5.6 nm at $800{ }^{\circ} \mathrm{C}$, given that $\boldsymbol{k}_{\boldsymbol{B}}$ is the Boltzmann constant, $T$ is temperature, $\Omega$ is the atomic volume of $\operatorname{Pt}\left(0.015 \mathrm{~nm}^{3}\right)$, and $\gamma$ is the surface tension of $\mathrm{Pt}$ (in the range of $1.4-2.8 \mathrm{~N} / \mathrm{m}$ ), ${ }^{26} v_{P}$ is the thermal vibration frequency of $\mathrm{Pt}$ atoms in the lattice (taken as $10^{14} \mathrm{~Hz}$ ), ${ }^{17} \theta$ is the contact angle between the Pt NP and the support (taken as $90^{\circ}$ ), $a$ is the Pt interatomic distance, and $E_{O R}$ is the activation energy of OR. The full equation was integrated numerically and fitted to the experimental data in Figure 3, allowing $\gamma$ and $E_{O R}$ to vary. Parameters and detailed fitting procedure are discussed in SI. The best fit, shown as the solid line in Figure S9, gave $K=9.6 \times 10^{-6} \mathrm{~nm}^{2}$ $\mathrm{s}^{-1}$ and $R=4.96 \mathrm{~nm}$, corresponding to an activation energy $\left(E_{O R}\right)$ of $364 \mathrm{~kJ} \mathrm{~mol}^{-1}$ and $\gamma=$ $2.45 \mathrm{~N} \mathrm{~m}^{-1}$. Conceptually, $E_{O R}$ can be expressed as $E_{O R}=\Delta H_{S}-E_{a d}+E_{d}$, where $\Delta H_{s}$ is the sublimation enthalpy of $\mathrm{Pt}\left(\sim 565 \mathrm{~kJ} \mathrm{~mol}^{-1}\right),{ }^{27} E_{a d}$ is the Pt atom adsorption energy ( 2-2.5 eV in vacuum), ${ }^{28}$ and $E_{d}$ is the energy barrier for Pt atoms to migrate on the alumina support $\left(\sim 0.2-0.5 \mathrm{eV}\right.$ in vacuum). ${ }^{28}$ Thus, the activation energy is within the range of 340-420 kJ mol${ }^{-1}$, which agrees with the activation energy extracted from our NP shrinking rate. Although the parameters used in this analysis may not be uniquely determined, the fact that they are at least reasonable provides confidence that it correctly captures the essential characteristics of OR. To further illustrate the effect of NP size on the phenomenon of OR, the initial NP shrinking rates for different combinations of $r$ and $r^{*}$, computed by Equation 1 with all the other parameters given above, are listed in Table 1. As shown in the table, the shrinking rate for a NP with diameter of $2 r=2 \mathrm{~nm}$ increases significantly as $2 r^{*}$ increases from $2.2 \mathrm{~nm}$ to 5 or $10 \mathrm{~nm}$. Because the equilibrium radius $\mathrm{r}^{*}$ can be estimated as an arithmetic mean radius in the interface controlled regime, the difference between $r$ and $r^{*}$ corresponds to the width of PSD, demonstrating that OR is suppressed when the PSD is relatively narrow. For larger NPs, with $2 r \mid 2 r *$ combinations of $5|10,5| 20$, and $5 \mid 50$, OR is not as prominent, due to the temperature dependence of the capillary length, $R$, and thus the width of the PSD is less relevant. These results clearly demonstrate how OR reflects the difference of surface energy, expressed as $2 \gamma \Omega / \mathrm{r}$, between small and large NPs, which drastically increases with decreasing size. Our observations are thus in agreement with previous findings that OR is a dominant NP growth mechanism in samples containing particles $2-5 \mathrm{~nm}$ in diameter. ${ }^{15}$

Histograms showing the evolution of the PSD of the mixed NP sample at $800{ }^{\circ} \mathrm{C}$, together with a summary plot of average NP size versus time, are shown in Figure 4 . The most significant change in the PSD occurs in the first $110 \mathrm{~min}$, and a change in slope is also found at $110 \mathrm{~min}$ in the plot of average NP size versus time, corresponding to a 
decreased growth rate for times greater than $110 \mathrm{~min}$. Since OR is the dominant mechanism, the decrease in growth rate may be compared with the NP shrinking rate that was derived above. At the beginning of the $800{ }^{\circ} \mathrm{C}$ portion of the experiment, taking an $r \mid r^{*}$ combination of $0.9 \mid 2$, we obtain an initial NP shrinking rate of $-2.5 \times 10^{-3} \mathrm{~nm} / \mathrm{s}$, while after 110 min, taking an $r \mid r^{*}$ combination of $1.2 \mid 2$, we obtain a rate of $-4.0 \times 10^{-4} \mathrm{~nm} / \mathrm{s}$, lower by $\sim 6$ times. We therefore propose that the decrease in growth rate with time is due to the fact that smaller particles, which are the primary driving force for the decrease of surface area, are depleted in the process of OR, therefore providing further support for this mechanism of deactivation.

Based on the comparison of tens of sequential images taken on the small sample, we found that no change in NP number and size could be discerned in regions of relatively low $\left(\sim 14 \times 10^{-4} \mathrm{~nm}^{-2}\right.$, detailed calculation of local areal density is shown in SI) density after the full $15 \mathrm{~h}$ experiment, as shown in Figure 5, thus confirming that OR has been drastically limited under these conditions. On the other hand, clear evidence of particle migration over distances of $\sim 20 \mathrm{~nm}$ was observed. Since this distance is much less than the inter-particle spacing in these low-density regions, coalescence could not occur, either. For example, the particles indicated by yellow arrows in Figure 5 were clearly diffusing around at $800{ }^{\circ} \mathrm{C}$, but they didn't shrink or coalesce with other particles nearby. However, in other regions with somewhat higher local NP density $\left(\sim 20 \times 10^{-4} \mathrm{~nm}^{-2}\right)$, PMC is clearly taking place, as indicated by the red circles in Figure 6. Calculation confirms that the additive volume of the four particles shown in Figure 6(f), for example, is equal to that of the single particle shown in Figure 6(h).

Since OR has been drastically limited in low density regions of the small (and large) NP samples, the slight shift to larger size observed in the small NP sample at $800{ }^{\circ} \mathrm{C}$ is mostly attributed to PMC. The overall process of PMC can be divided into two steps, random migration followed by collision-induced coalescence. ${ }^{24}$ Since the time required for coalescence is much shorter than that needed for migration in this case, particle migration is the rate-controlling step. It arises from the random motion of the supported NPs due to the rapid atomic diffusion on their surfaces. The mobility of supported NPs thus decreases rapidly with increasing NP size. ${ }^{24}$ By taking $20 \mathrm{~nm}$ as an average migration distance $\left(2 \sqrt{D_{N P} t}\right)$ of the small NPs, based on a series of sequential images recorded over a $5 \mathrm{~h}$ period at $800{ }^{\circ} \mathrm{C}$, we deduce that the particle diffusion coefficient, $D_{N P}$, is on the order of $10^{-17} \mathrm{~cm}^{2} \mathrm{~s}^{-1}$. In the classical model, $D_{N P}$ can be expressed as $0.301 D_{s}\left(\frac{a}{r}\right)^{4}$ for spherical particles, where $D_{s}$ is the surface atom diffusion coefficient, $r$ is the NP radius, and $a$ is the atomic spacing. ${ }^{24}$ Surprisingly, the measured $D_{N P}$ is 5 orders of magnitude smaller than the classical model predicts. ${ }^{24,29}$ We speculate that the large discrepancy may be due to the geometry of the support and the specific bonding between the alumina support and the Pt NPs, also observed in other similar systems. ${ }^{18,30}$ The contribution of PMC to particle coarsening in this case is thus much smaller than expected. The strong dependence of $D_{N P}$ on the NP size further implies that the large particles are almost immobile (since the calculated migration distance is less than the diameter), and this was indeed confirmed by sequential HAADF images, such as those shown in Figure 7. Although the size dependence of the particle diffusion coefficient can provide useful knowledge in controlling the PMC rate through optimization of particle 
loading, further investigation of particle diffusion in real catalysts is clearly needed, given the discrepancy between experiment and theory revealed here.

\section{CONCLUSIONS}

In summary, through a unique combination of novel in-situ atomic resolution transmission electron microscopy performed at elevated temperatures under atmospheric pressure conditions on size selected Pt NPs supported on high-surface-area $\mathrm{Al}_{2} \mathrm{O}_{3}$, we have been able to cleanly separate and individually characterize the processes of OR and PMC under reducing conditions. Further, our work suggests that OR can be efficiently limited through the use of monodisperse NPs with narrow PSD in real catalysts, though fluctuations limit the time over which this situation persists, as shown by recent Monte Carlo simulations. ${ }^{31}$ The contribution of PMC was found to be much smaller than expected, based on the classical model for particle diffusion coefficient. In principle, it should be possible to also limit PMC by the appropriate choice of NP size. We therefore provide useful general guidelines for the preservation of high catalyst dispersion with extended time in use.

\section{ASSOCIATED CONTENT}

\section{Supporting Information}

Movie (avi) together with materials, method and Figures S1-S10. This material is available free of charge via the Internet at http://pubs.acs.org."

\section{ACKNOWLEDGMENT}

The work at the University of Michigan was supported by the National Science Foundation under grants CBET1159240 and DMR-0723032. M.C. acknowledges support from the National Science Foundation through the Nano/Bio Interface Center at the University of Pennsylvania, Grant DMR-0832802. C.B.M. is grateful for the support of the Richard Perry University Professorship. 
Table 1 Initial NP shrinking rates $(\mathrm{dr} / \mathrm{dt})$ for various combinations of $2 r$ and $2 r$ *

\begin{tabular}{|c|c|c|c|c|c|c|}
\hline $2 r \mid 2 r^{*}$ & $2 \mid 2.2$ & $2 \mid 5$ & $2 \mid 10$ & $5 \mid 10$ & $5 \mid 20$ & $5 \mid 50$ \\
\hline $\begin{array}{c}d r / d t \\
\left(10^{-4} \mathrm{~nm} / \mathrm{s}\right)\end{array}$ & -5.0 & -13 & -13.4 & -0.17 & -0.22 & -0.23 \\
\hline
\end{tabular}




\section{REFERENCES}

(1) Gates, B. Chem. Rev. 1995, 95, 511-522.

(2) Bunluesin, T.; Gorte, R.; Graham, G. Appl. Catal., B. 1998, 15, 107-114.

(3) Campbell, C. T.; Parker, S. C.; Starr, D. E. Science 2002, 298, 811-814.

(4) Galvis, H. M. T.; Bitter, J. H.; Khare, C. B.; Ruitenbeek, M.; Dugulan, A. I.; de Jong, K. P. Science 2012, 335, 835-838.

(5) Cargnello, M.; Doan-Nguyen, V. V.; Gordon, T. R.; Diaz, R. E.; Stach, E. A.; Gorte, R. J.; Fornasiero, P.; Murray, C. B. Science 2013, 341, 771-773.

(6) Tian, Y.; Tatsuma, T. Chem. Commun. 2004, 1810-1811.

(7) Lu, J.; Do, I.; Drzal, L. T.; Worden, R. M.; Lee, I. ACS nano 2008, 2, 1825-1832.

(8) Joo, S. H.; Choi, S. J.; Oh, I.; Kwak, J.; Liu, Z.; Terasaki, O.; Ryoo, R. Nature 2001, 412, 169-172.

(9) Liu, Z.; Ling, X. Y.; Su, X.; Lee, J. Y. J. Phys. Chem. B 2004, 108, 8234-8240.

(10) Alayoglu, S.; Nilekar, A. U.; Mavrikakis, M.; Eichhorn, B. Nat. Mater. 2008, 7, 333-338.

(11) Aricò, A. S.; Bruce, P.; Scrosati, B.; Tarascon, J.-M.; Van Schalkwijk, W. Nat. Mater. 2005, 4, 366-377.

(12) White, R. J.; Luque, R.; Budarin, V. L.; Clark, J. H.; Macquarrie, D. J. Chem. Soc. Rev. 2009, 38, 481-494.

(13) Datye, A. K.; Xu, Q.; Kharas, K. C.; McCarty, J. M. Catal. Today 2006, 111, 5967.

(14) DeLaRiva, A. T.; Hansen, T. W.; Challa, S. R.; Datye, A. K. J. Catal. 2013, 308, 291-305.

(15) Hansen, T. W.; DeLaRiva, A. T.; Challa, S. R.; Datye, A. K. Acc. Chem. Res. 2012, 46, 1720-1730.

(16) Behafarid, F.; Pandey, S.; Diaz, R. E.; Stach, E. A.; Cuenya, B. R. Phys. Chem. Chem. Phys. 2014, 16, 18176-84.

(17) Challa, S. R.; Delariva, A. T.; Hansen, T. W.; Helveg, S.; Sehested, J.; Hansen, P. L.; Garzon, F.; Datye, A. K. J. Am. Chem. Soc. 2011, 133, 20672-5.

(18) Simonsen, S. B.; Chorkendorff, I.; Dahl, S.; Skoglundh, M.; Sehested, J.; Helveg, S. J. Am. Chem. Soc. 2010, 132, 7968-7975.

(19) Allard, L. F.; Wittig, J. E.; Chi, M.; More, K. L.; Bigelow, W. C.; Damiano, J.; Nackashi, D. P. Microsc. Microanal. 2013, 19, 1474.

(20) Zhang, S.; Chen, C.; Cargnello, M.; Fornasiero, P.; Gorte, R. J.; Graham, G. W.; Pan, X. Nat. Commun. 2015, 6, 7778. 
(21) Vendelbo, S.; Elkjær, C.; Falsig, H.; Puspitasari, I.; Dona, P.; Mele, L.; Morana, B.; Nelissen, B.; van Rijn, R.; Creemer, J.; Kooyman, P. J.; Helveg, S. Nat. Mater. 2014 13 884-890.

(22). Wettergren, K.; Schweinberger, F. F.; Deiana, D.; Ridge, C. J.; Crampton, A. S.; Rotzer, M. D.; Hansen, T. W.; Zhdanov, V. P.; Heiz, U.; Langhammer, C. Nano Lett. 2014, 14, 5803-5809.

(23) Cargnello, M.; Chen, C.; Diroll, B. T.; Doan-Nguyen, V. V.; Gorte, R. J.; Murray, C. B. J. Am. Chem. Soc. 2015, 137, 6906-6911.

(24). Wynblatt, P.; Gjostein, N. Prog. Solid State Chem. 1975, 9, 21-58.

(25). Houk, L. R.; Challa, S. R.; Grayson, B.; Fanson, P.; Datye, A. K. Langmuir 2009, $11225-11227$.

(26) Overbury, S.; Bertrand, P.; Somorjai, G. Chem. Rev. 1975, 75, 547-560.

(27) Arblaster, J. W. Platinum Met. Rev. 2005, 49, 141-149.

(28) Aaron Deskins, N.; Mei, D.; Dupuis, M. Surf. Sci. 2009, 603, 2793-2807.

(29) Schumacher, D.; Seeger, A.; Härlin, O. Phys Status Solidi B 1968, 25, 359-371.

(30) Mei, D.; Kwak, J. H.; Hu, J.; Cho, S. J.; Szanyi, J.; Allard, L. F.; Peden, C. H. J. Phys. Chem. Lett. 2010, 1, 2688-2691.

(31) Zhdanov, V. P.; Schweinberger, F. F.; Heiz, U.; Langhammer, C. Chem. Phys. Lett. 2015, 631, 21-25. 


\section{Figure Captions:}

Figure 1 HAADF images of the as-prepared mixed NP sample (a) and individual large (b) and small (c) Pt NPs.

Figure 2 Pt PSDs of the small (a), large (b) and mixed (c) NP samples obtained from HAADF images taken during in-situ observation at the indicated temperatures. The areato-volume ratios $(\mathrm{A} / \mathrm{V})$, assuming hemispherical NPs, computed from initial and final PSDs, are also shown for each sample.

Figure 3 Sequential images showing the gradual shrinking and disappearance of the small NPs in the mixed NP sample. Elapsed time, in seconds, is indicated on the top of each HAADF image, taken at $800{ }^{\circ} \mathrm{C}$. A plot showing the radius of four NPs as a function of time is shown in the bottom left. The dashed line corresponds to the value of $r^{*}$ used in fitting, as discussed in SI. The error bar for all points is $\pm 0.07 \mathrm{~nm}$, as shown on the plot.

Figure 4. Pt PSDs of the mixed NP sample obtained after 0, $50 \mathrm{~min}, 110 \mathrm{~min}, 180 \mathrm{~min}$, and $300 \mathrm{~min}$ from in-situ observations during heating at $800{ }^{\circ} \mathrm{C}$. The time dependence of average NP size is shown in the bottom right panel.

Figure 5 Sequential HAADF images showing an area of the small NP sample with relatively low $\left(14 \times 10^{-4} \mathrm{~nm}^{-2}\right) \mathrm{NP}$ density. (a-d) were acquired at 0 (at the end of $700{ }^{\circ} \mathrm{C}$ ), $1,3.5$ and 5 hours at $800{ }^{\circ} \mathrm{C}$.

Figure 6 Sequential HAADF images (a, c, e, g) and zoom-in areas (b, d, f, h) indicated by the red circles showing an area of the small NP sample with relatively high $\left(20 \times 10^{-4}\right.$ $\mathrm{nm}^{-2}$ ) NP density. (a,c,e,g) were acquired at 0 (at the end of $700{ }^{\circ} \mathrm{C}$ ), 1, 3.5, and 5 hours at $800{ }^{\circ} \mathrm{C}$.

Figure 7 Sequential HAADF images showing an area of the large NP sample at initial stage (a), at the end of $700{ }^{\circ} \mathrm{C}$ (b) and $800{ }^{\circ} \mathrm{C}$ (c) aging. 
FIGURES

Figure 1.

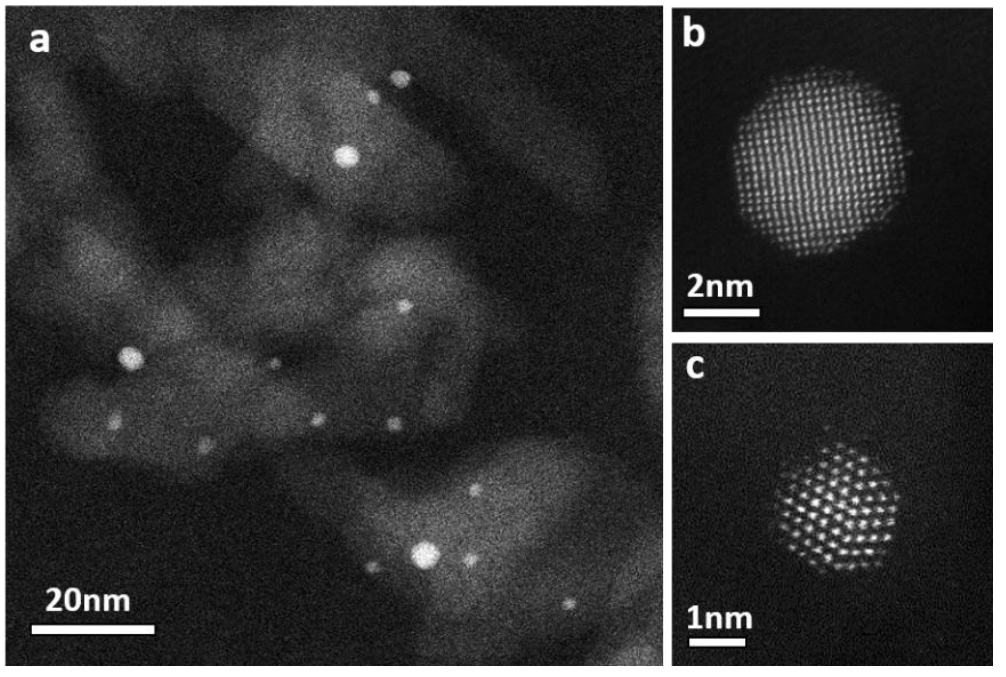


Figure 2.

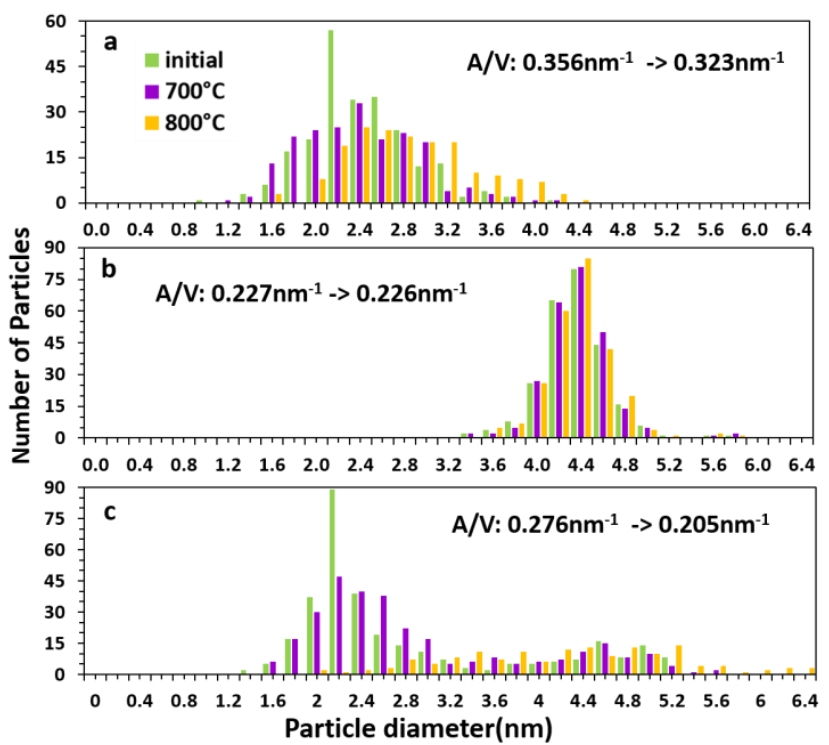


Figure 3.

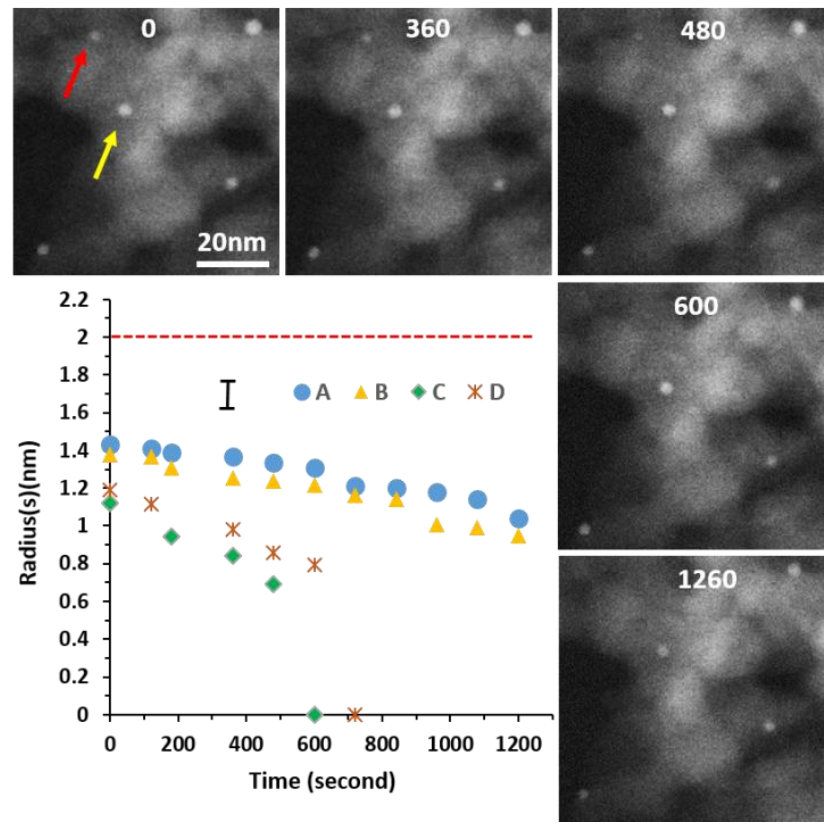


Figure 4.

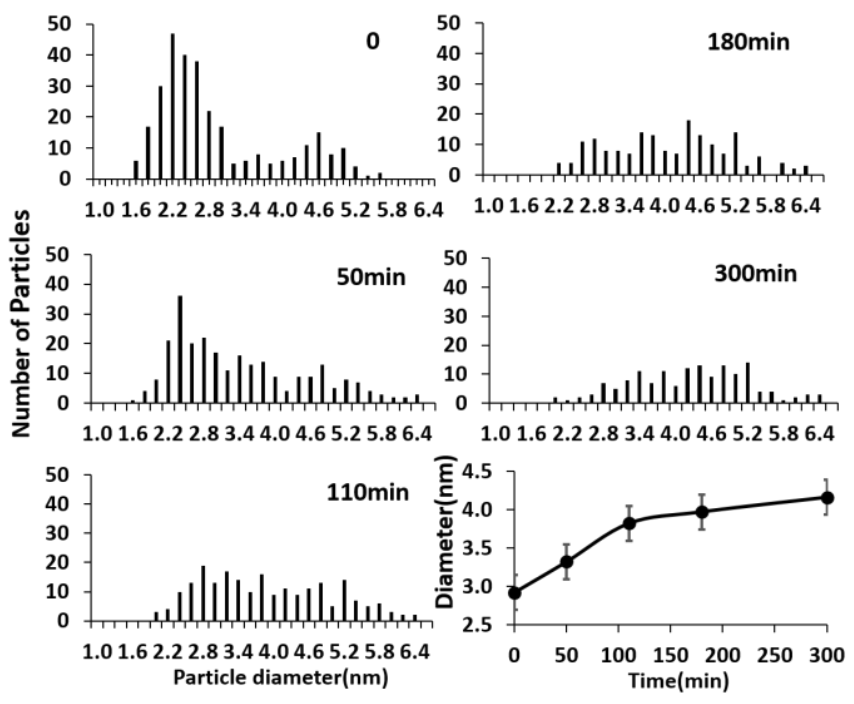


Figure 5.

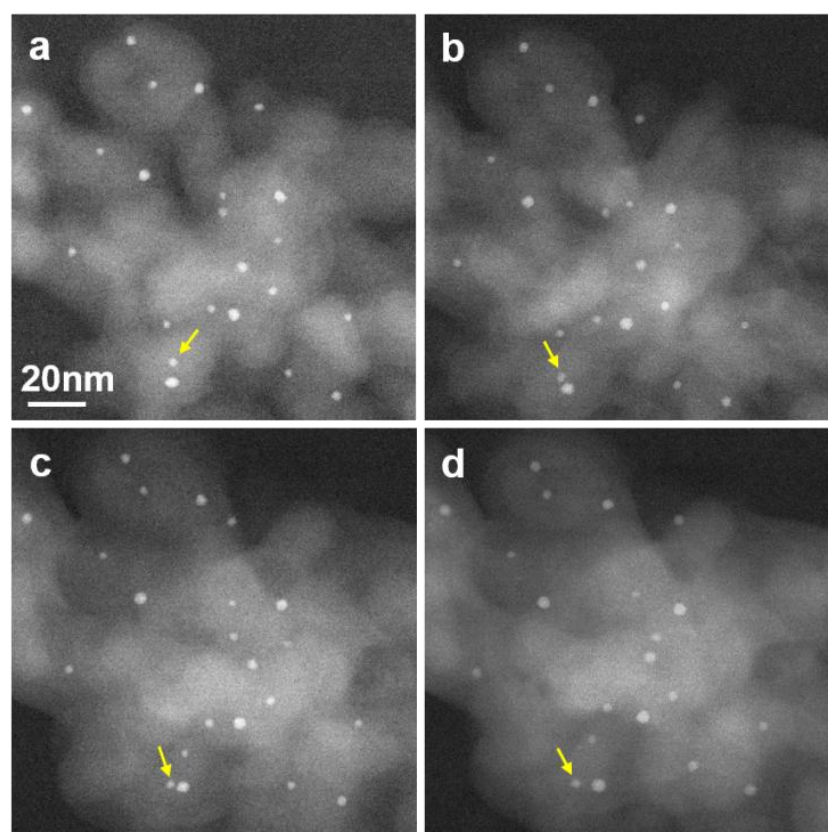




\section{Figure 6.}

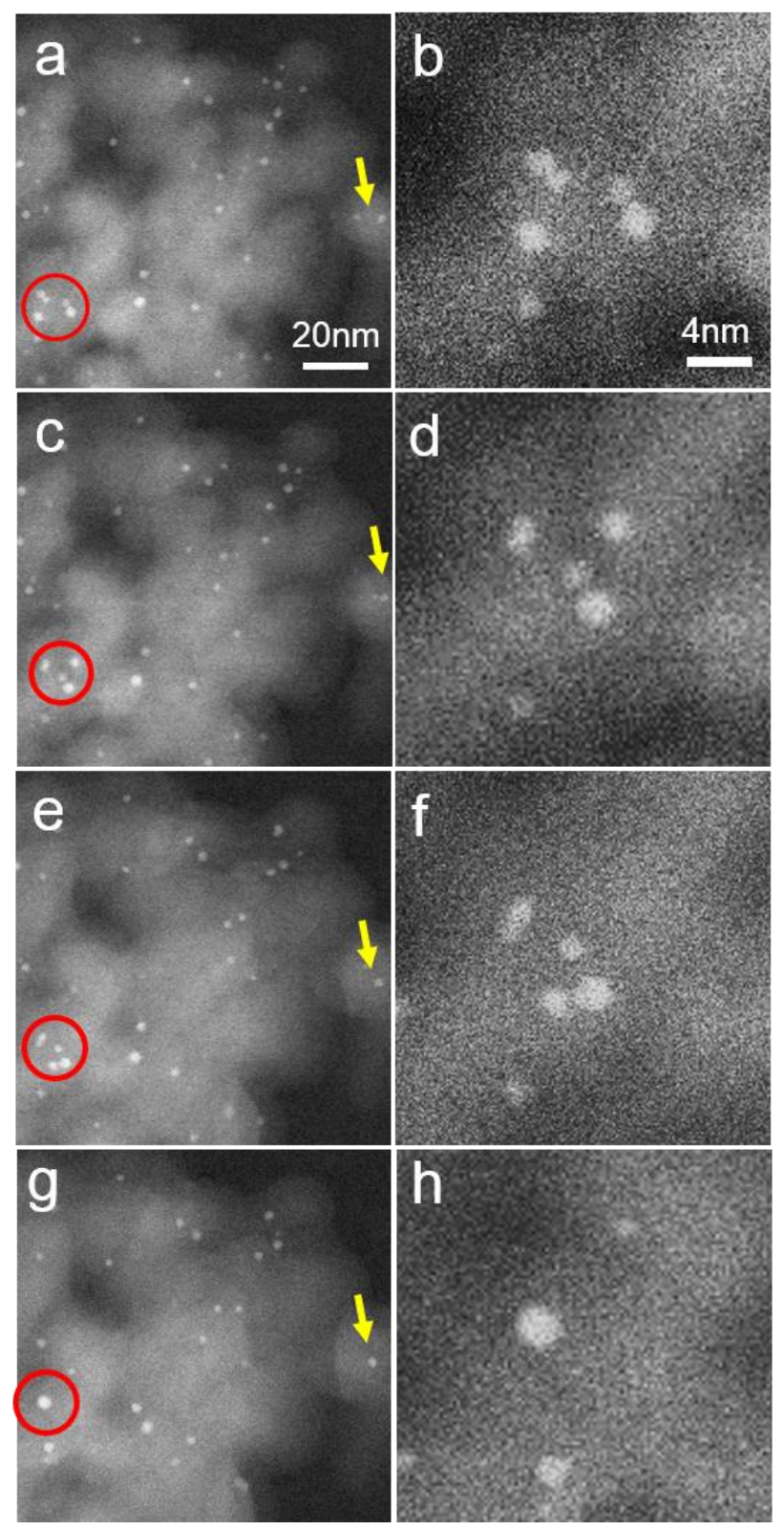


Figure 7.
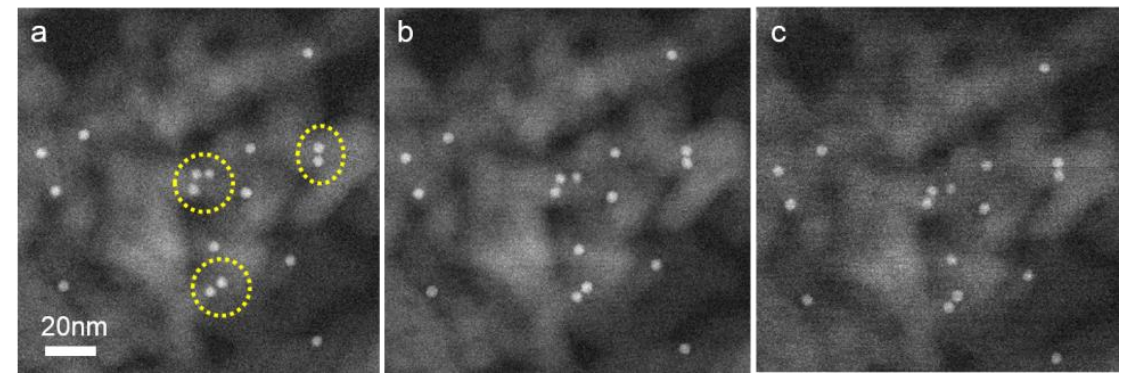
$0.5 w \% P t / \mathrm{Al}_{2} \mathrm{O}_{3}$ in 760 Torr $\% 5 \mathrm{H}_{2} / \mathrm{N}_{2}$ at $800{ }^{\circ} \mathrm{C}$

Ostwald Ripening $+$

Migration/Coalescence

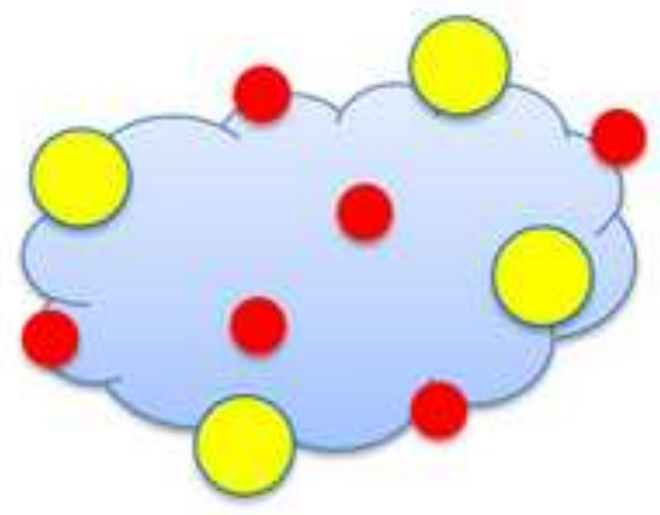

Migration/Coalescence

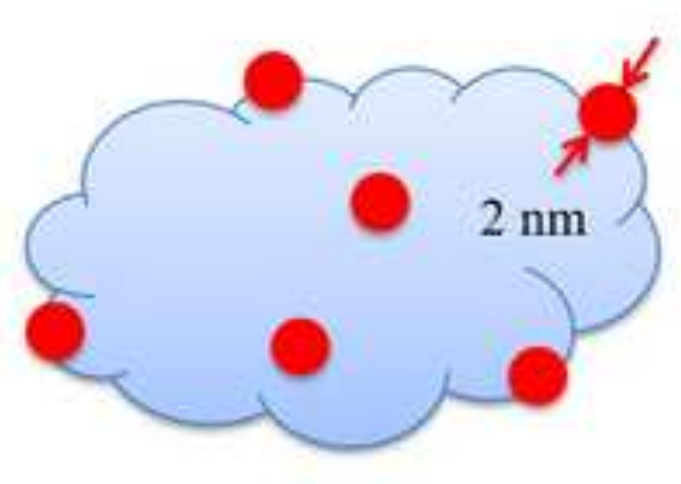

No Growth

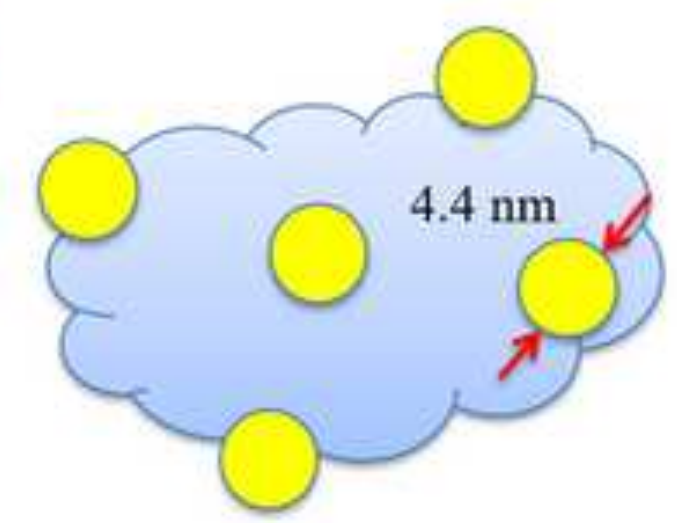

